\title{
LIVER
}

\section{Hepatic triglyceride content and its relation to body adiposity: a magnetic resonance imaging and proton magnetic resonance spectroscopy study}

\section{E L Thomas, G Hamilton, N Patel, R O'Dwyer, C J Doré, R D Goldin, J D Bell, S D Taylor-Robinson}

See end of article for authors' affiliations

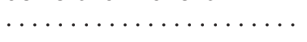

Correspondence to: Dr J D Bell, MRI Unit, Hammersmith Hospital, Du Cane Rd, London W12 OHS, UK; ;immy.bell@ csc.mrc.ac.uk

Revised version received 24 May 2004

Accepted for publication 25 May 2004
Background: Hepatic steatosis is associated with obesity and type II diabetes. Proton magnetic resonance spectroscopy ( ${ }^{1} \mathrm{H}$ MRS) is a non-invasive method for measurement of tissue fat content, including intrahepatocellular lipids (IHCL) and intramyocellular lipids (IMCL).

Patients and methods: We used ' $\mathrm{H}$ MRS and whole body magnetic resonance imaging (MRI) to assess the relationship between IHCL accumulation, total body adipose tissue (AT) content/distribution, and IMCL content in 11 subjects with biopsy proven hepatic steatosis and 23 normal volunteers.

Results: $\mathrm{HCL}$ signals were detectable in all subjects but were significantly greater in hepatic steatosis (geometric mean (GM) 11.5 (interquartile range (IQR) 7.0-39.0)) than in normal volunteers (GM 2.7 (IQR 0.7-9.3); $p=0.02$ ). In the study group as a whole, IHCL levels were significantly greater in overweight compared with lean subjects (body mass index (BMI) $>25 \mathrm{~kg} / \mathrm{m}^{2}(\mathrm{n}=23)$ : GM 7.7 (IQR 4.0-28.6) v BMI $<25 \mathrm{~kg} / \mathrm{m}^{2}(\mathrm{n}=11)$ : GM 1.3 (IQR 0.3-3.6; $\left.\left.\mathrm{p}=0.004\right)\right)$. There was a significant association between IHCL content and indices of overall obesity (expressed as a percentage of body weight) for total body fat $(p=0.001)$, total subcutaneous AT $(p=0.007)$, and central obesity (subcutaneous abdominal AT $(p=0.001)$ and intra-abdominal AT $(p=0.001))$, after allowing for sex and age. No correlation between $\mathrm{IHCL}$ content and IMCL was observed. A significant correlation was observed between serum alanine aminotransferase and liver fat content $(r=0.57, p=0.006)$.

Conclusions: Our results suggest that hepatic steatosis appears to be closely related to body adiposity, especially central obesity. MRS may be a useful method for monitoring $\mathrm{IHCL}$ in future interventional studies.
$\mathrm{F}$ at accumulation in the liver is commonly associated with obesity, insulin resistance, and type II diabetes, although the mechanism underlying this relationship is not fully understood..$^{1-7}$

Until recently, the sole method available for quantification of intrahepatocellular lipid (IHCL) content was through the use of liver biopsy techniques. ${ }^{8}$ However, this procedure has many drawbacks, even when performed under ultrasound guidance, including discomfort due to its invasive nature, risk of infection, haematoma formation or more significant internal bleeding, and potentially biliary leakage. These factors make the use of percutaneous biopsy ethically difficult in a research context and impractical for longitudinal studies. Furthermore, biopsies are subject to sampling error, providing quantitative information on a rather small volume ( $\sim 0.01-0.05 \mathrm{ml})$ of liver, which may be of limited use in the presence of heterogeneous IHCL distribution. Ultrasound, computerised tomography (CT), and magnetic resonance imaging (MRI) can be used to detect fatty liver but are not routinely used to provide quantitative information of fat content, although methods have been developed for using CT to assess hepatic fat in a quantitative manner. ${ }^{9}{ }^{10}$ However, repeated CT scanning has significant associated radiation exposure and thus is inappropriate in interventional studies where serial measurements may be required. More recently, proton magnetic resonance spectroscopy $\left({ }^{1} \mathrm{H}\right.$ MRS $)$ has been shown to be a fast, safe, non-invasive method for the relative quantification of IHCL content. ${ }^{11-20}$ This imaging modality should enable the true significance of IHCL accumulation in the pathogenesis of conditions such as insulin resistance and type II diabetes to be understood. ${ }^{12}$
Although the use of ${ }^{1} \mathrm{H}$ MRS to quantify IHCL deposition is becoming more widely used, a number of important issues still remain unresolved, including the relationship between IHCL and other body fat depots and the impact of environmental and genetic factors. Furthermore, studies in cadavers $^{21}$ suggest that the distribution of lipid in the human liver is non-homogeneous. ${ }^{22}$

In the present study, we have utilised localised hepatic and calf muscle ${ }^{1} \mathrm{H}$ MRS, in conjunction with whole body MRI, to determine the relationship between IHCL accumulation and whole body adipose tissue content and distribution in a cohort of subjects with biopsy proven non-alcoholic fatty liver and also in subjects from the general population without a history of liver or metabolic disease. The results suggest that IHCL accumulation is widespread and appears to be closely related to abdominal fat adiposity.

\section{METHODS}

Written informed consent was obtained from all volunteers. Permission for this study was obtained from the Ethics Committee of Hammersmith Hospital, Imperial College London (Rec. 93/4047; 93/3995). The study protocol conformed to the ethical guidelines of the 1975 Declaration of
Abbreviations: ALP, alkaline phosphatase; ALT, alanine aminotransferase; $\gamma \mathrm{GT}, \gamma$ glutamyl transferase; $\mathrm{AT}$, adipose tissue; BMI, body mass index; CoV, coefficient of variation; CT, computerised tomography; $\mathrm{GM}$, geometric mean; $\mathrm{HCL}$, intrahepatocellular lipids; $I M C L$, intramyocellular lipids; IQR, interquartile range; LFTs, liver function tests; MRI, magnetic resonance imaging; ${ }^{1} \mathrm{H}$ MRS, proton magnetic resonance spectroscopy; NASH, non-alcoholic steatohepatitis; $T E$, echo time; $T R$, repetition time 
Table 1 Population characteristics and differences between non-alcoholic steatohepatitis (NASH) patients and controls in hepatic fat and body adiposity

\begin{tabular}{|c|c|c|c|}
\hline & $\begin{array}{l}\text { Controls } \\
(n=23)\end{array}$ & $\begin{array}{l}\text { NASH } \\
(n=11)\end{array}$ & p Value \\
\hline $\mathrm{IHCL}^{*}$ & $2.7[0.7-9.3]$ & $11.5[7.0-39.0]$ & 0.02 \\
\hline Liver volume* & $1.7[1.5-1.9]$ & $1.8[1.5-2.1]$ & 0.2 \\
\hline Age $(y)$ & $43.1(11.9)$ & $52.4(11.9)$ & 0.04 \\
\hline $\operatorname{Sex}(F / M)$ & $6 / 17$ & $4 / 7$ & 0.7 \\
\hline Height (m) & $1.72(0.08)$ & $1.72(0.12)$ & 0.9 \\
\hline Weight (kg) & $80.8(13.9)$ & $85.9(16.1)$ & 0.3 \\
\hline BMI $\left(\mathrm{kg} / \mathrm{m}^{2}\right)$ & $27.2(4.1)$ & $29.0(5.0)$ & 0.3 \\
\hline$\%$ Body AT $\dagger$ & $34.0(6.9)$ & $38.5(6.1)$ & 0.08 \\
\hline \% Subcutaneous AT $\ddagger$ & $24.9(7.2)$ & $28.3(6.3)$ & 0.2 \\
\hline \% Subcutaneous abdominal AT & $6.7(2.7)$ & $7.8(2.5)$ & 0.3 \\
\hline \% Subcutaneous peripheral AT & $18.2(4.6)$ & $20.5(4.1)$ & 0.17 \\
\hline$\%$ Internal AT & $9.1(2.6)$ & $10.2(1.4)$ & 0.19 \\
\hline$\%$ Intra-abdominal AT & $5.1(1.9)$ & $5.6(0.9)$ & 0.4 \\
\hline$\%$ Non-abdominal internal AT $\ddagger$ & $4.0(0.9)$ & $4.6(1.0)$ & 0.08 \\
\hline Soleus IMCL* & 19 [13-30] & $21[13-30]$ & 0.5 \\
\hline Tibialis IMCL & $8.2(4.3)$ & $10.4(4.2)$ & 0.17 \\
\hline$\gamma \mathrm{GT}^{*}$ & 47 [29-75] & $74[27-212]$ & 0.3 \\
\hline $\mathrm{ALT}^{*}$ & $36[23-56]$ & 89 [74-122] & $<0.001$ \\
\hline ALP* & 60 [54-68] & $81[72-98]$ & 0.007 \\
\hline
\end{tabular}

Summary measures are mean (SD).

*Statistical analysis performed on $\log _{\mathrm{e}}$ transformed variables, summary measures are geometric mean [interquartile range].

tWith reference to total body mass.

WWith reference to total adipose tissue.

IHCL, intrahepatocellular lipids; BMI, body mass index; AT, adipose tissue; IMCL, intramyocellular lipids; ALP,

alkaline phosphatase; ALT, alanine aminotransferase; $\gamma \mathrm{GT}$, gamma-glutamyl transferase.

Helsinki. A cohort of 34 fasted volunteers was studied (table 1). Eleven (four females, seven males) had previously been diagnosed as having hepatic steatosis following liver biopsy; the remaining 23 (six females and 17 males) were healthy volunteers from the general population without a prior history of liver or metabolic disease. These healthy volunteers were recruited from the hospital staff or were friends or relatives of the hospital staff. None had abnormal liver biochemistry. No patient or volunteer drank alcohol in excess of $20 \mathrm{~g} /$ day and none had a history of alcohol misuse.

Of the 11 patients who had undergone liver biopsy within the previous six months for formal assessment of nonalcoholic steatohepatitis (NASH), all had persistently abnormal liver function tests (LFTs) with elevated serum levels of alanine aminotransferase (ALT: geometric mean 49.0 (interquartile range (IQR) 28-93) IU/l) and/or serum $\gamma$ glutamyl transferase ( $\gamma$ GT: geometric mean 55.1 (IQR 29-77) IU/l). Alkaline phosphatase levels were also measured (ALP: geometric mean 66.4 (IQR 59-75) U/1). One patient had type II diabetes, six had dyslipidaemia, and four were simply overweight. All liver biopsies were examined by an experienced histopathologist (RG) using the Brunt's scoring system, which includes grading (hepatic steatosis, hepatic ballooning, portal and intra-acinar inflammation) and staging the degree of fibrosis. ${ }^{23}$ All had evidence of hepatic steatosis on histological examination (grade 1 (mild steatosis) to grade 3 (severe steatosis)), four had evidence of mild inflammation (grade 1-2 intra-acinar and/or portal inflammation), and three had fibrosis (stage 1-3), but not cirrhosis. The clinical details of the patient and volunteer populations are detailed in table 1.

\section{MRI of the liver}

Imaging data were acquired with a $1.5 \mathrm{~T}$ Eclipse multinuclear system (Philips Medical Systems, Cleveland, Ohio) using a $45 \mathrm{~cm}$ diameter elliptical body coil, a $14 \mathrm{~cm}$ diameter surface coil, and a $\mathrm{T}_{1}$ weighted spin echo sequence with a repetition time (TR) of $800 \mathrm{~ms}$, echo time (TE) of $16 \mathrm{~ms}$, and four signal averages. Between 25 and 35 (depending on liver size from pilot images) $6 \mathrm{~mm}$ thick transverse images were acquired per subject. Images were analysed using an image segmentation software program in which liver contours were manually drawn for each slice. ${ }^{24}$ Liver volumes were calculated from this by multiplying the liver tissue volume of each slice by slice thickness (6 $\mathrm{mm})$.

\section{MRS of the liver}

${ }^{1} \mathrm{H}$ MR spectra were acquired at $1.5 \mathrm{~T}$ from the right lobe of the liver using a PRESS sequence (TR $1500 \mathrm{~ms} / \mathrm{TE} 135 \mathrm{~ms}$ ) without water saturation and with 128 signal averages. Transverse images of the liver were used to ensure accurate positioning of the $(20 \times 20 \times 20 \mathrm{~mm})$ voxel in the liver, avoiding blood vessels, the gall bladder, and fatty tissue. Spectra were analysed in the time domain using the AMARES algorithm included in the MRUI software package. ${ }^{25}{ }^{26}$ Peak areas for all resonances were obtained and lipid resonances were quantified with reference to water resonance, after correcting for $T_{1}$ and $T_{2}$. These $T_{1}$ and $T_{2}$ values for IHCL were obtained by an experienced physicist (GH). The following values were obtained: $\mathrm{T}_{1}$ of the $\mathrm{CH}_{2}$ resonance of IHCL $=480 \mathrm{~ms} ; \mathrm{T}_{1}$ of the water resonance $=1230 \mathrm{~ms} ; \mathrm{T}_{2}$ of the $\mathrm{CH}_{2}$ resonance of $\mathrm{IHCL}=50 \mathrm{~ms} ; \mathrm{T}_{2}$ of the water resonance $=36 \mathrm{~ms}$.

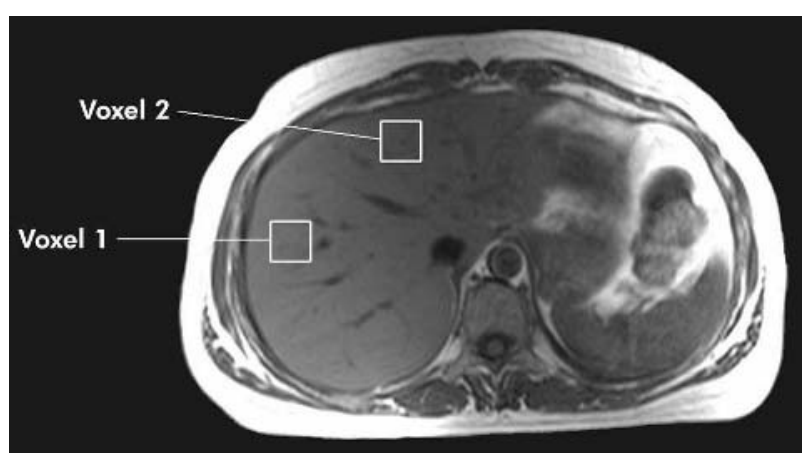

Figure 1 A transverse image through the abdomen showing the two voxel positions used to study regional variation in hepatic fat content. 
Results are expressed as percentage ratio of the $\mathrm{CH}_{2}$ lipid peak area relative to the water peak area.

To assess the reproducibility of the ${ }^{1} \mathrm{H}$ MRS results, analyses were repeated in triplicate and the coefficient of variation measured. Intra-examination reproducibility was measured by acquiring three voxels in the same position in a single examination while inter-examination reproducibility was assessed by acquiring a voxel from the same position in the liver, in each given subject, on three different occasions.

To determine potential regional lipid variation within the liver, ${ }^{1} \mathrm{H}$ MR spectra were obtained in 12 volunteers by positioning a same sized voxel in two different positions within the right lobe of the liver (fig 1).

\section{MRS of muscle}

Proton MR spectra were acquired from $20 \times 20 \times 20 \mathrm{~mm}$ voxels localised to the soleus and tibialis muscles of the left calf using a PRESS sequence (TR 1500 ms / TE 135 ms) with 256 signal averages, as previously described..$^{27}$ As in the liver, analysis was carried out using the AMARES algorithm in the MRUI software package. ${ }^{25} 26$ Peak areas for all resonances were obtained and lipid resonances were quantified with reference to total creatine after correcting for $T_{1}$ and $T_{2}$.

\section{Total body adipose tissue (AT) content}

Rapid $\mathrm{T}_{1}$ weighted MR images (TR $36 \mathrm{~ms}$, TE $14 \mathrm{~ms}$ ) were acquired on a Philips 1.0 T HPQ system, as previously described ${ }^{28}$ All images were collected at isocentre to avoid image distortion with the patient, in a prone position, being moved through the magnet to acquire full body coverage. A validated semiautomatic "in-house" program was then used to segment and analyse the AT. ${ }^{28}$ Total body AT, subcutaneous, total internal, subcutaneous abdominal, and intraabdominal AT volumes were measured.

\section{Biochemistry}

Blood was drawn on the same day as the initial MR scan for serum ALT, ALP, and $\gamma$ GT measurements, as recommended by the European Committee for Clinical Laboratory Standards.

\section{Statistical analysis}

Normally distributed results are expressed as mean (SD). $\log _{e}$ transformation was used for IHCL, liver volume, and

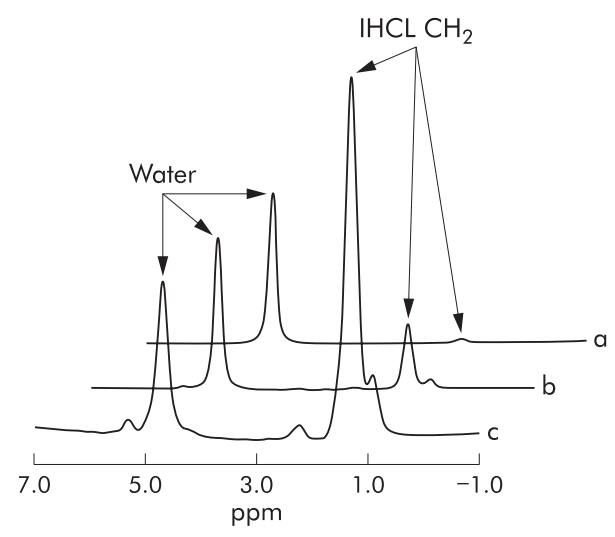

Figure 2 Typical proton magnetic resonance liver spectra from three volunteers showing progressive degrees of fatty infiltration. Spectrum (a) shows a liver with minimal fatty infiltration (1.0\%), (b) a liver with moderate fatty infiltration (10.2\%), and spectrum (c) shows a liver with severe fatty infiltration (74.9\%). Resonances from water and IHCL$\left(\mathrm{CH}_{2}\right)_{n}$ - can be clearly identified. Values refer to the peak area of the IHCL peak with reference to the water peak after correcting for $T_{1}$ and $T_{2}$. $\mathrm{IHCL}$, intrahepatocellular lipids. triglyceride levels in the liver and soleus muscle as their distribution was positively skewed. Results for log transformed variables are presented as geometric mean (GM) and interquartile range (IQR). A two sample $t$ test was used for comparisons of two independent mean values. The relationship between IHCL, liver volume, and individual AT depots was examined using multiple regression analysis to assess the independent effects of regional adiposity. Multiple regression analysis was performed to predict liver fat from the percentage fat in each compartment after adjusting for age and sex. The level of significance was set at 5\%. Data were analysed by CD using Stata (StataCorp 2001 Stata Statistical Software, Release 7.0; Stata Corporation, College Station, Texas, USA).

\section{RESULTS}

Results of the liver MRS, muscle MRS, whole body AT content measurement, and blood biochemistry in the NASH and control groups are summarised in table 1 .

\section{'H MRS of the liver}

Typical ${ }^{1} \mathrm{H}$ MR spectra from the liver of subjects with different degrees of fatty infiltration are shown in fig 2. IHCL was detectable by MRS in all of these subjects. Hepatic water was used as an internal standard, giving a geometric mean IHCL $\mathrm{CH}_{2} /$ water content for this cohort of subjects of 11.5 (IQR 7.0-39.0, range 1.3-74.9).

To assess the presence of IHCL accumulation in otherwise normal individuals without a history of abnormal liver function tests, ${ }^{1} \mathrm{H}$ MR spectra were obtained from 23 healthy volunteers with no known history of metabolic or liver disease. IHCL was detectable in all 23 subjects, with no significant differences between male and female volunteers (male GM 4.7 (IQR 1.8-10.8) v female GM 3.7 (IQR 0.328.6); $\mathrm{p}=0.72$ ). However, the geometric mean content for these subjects, separately or together, was significantly lower (2.7 (IQR 0.7-9.3, range 0.2-77.4); $\mathrm{p}=0.02$ ) than for those with biopsy proven fatty liver infiltration (table 1). This difference was not related to potential differences in MR relaxation characteristics $\left(\mathrm{T}_{1}\right.$ and $\left.\mathrm{T}_{2}\right)$ in these subjects, as increasing the repetition time ( $\mathrm{TR}=20 \mathrm{~s}$ ) or decreasing echo time ( $\mathrm{TE}=40 \mathrm{~ms}$ ) had little effect on the observed interindividual differences (results not shown).

\section{Reproducibility}

Mean inter-examination coefficient of variation $(\mathrm{CoV})$ for IHCL $\mathrm{CH}_{2}$ /water examination, assessed by taking the subject in and out of the scanner several times, then repeating imaging and repositioning the voxel each time, was found to be $7.0 \%$ (range $4.0-11.7 \%$ ). As expected, the mean intraexamination $\mathrm{CoV}$ was significantly lower $(6.0 \%)$.

\section{Heterogeneity of liver fat}

In a randomly selected subcohort of 12 subjects, ${ }^{1} \mathrm{H}$ MR spectra were obtained from two different locations within the right lobe of the liver. Overall, there appeared to be no significant regional differences in relative levels of IHCL although there was substantial interindividual variation, with some volunteers having a difference in fat content of up to $50 \%$ between regions whereas in others it was less than $1 \%$ (fig 3). This variation in fat content did not appear to be related to anatomy or the relative amount of fat present in the liver.

\section{Liver volumes}

Liver volumes were obtained from the MRI images acquired during the examination and ranged in size from 1.36 to $3.0 \mathrm{l}$. To evaluate the reproducibility of liver volumes, each volume 


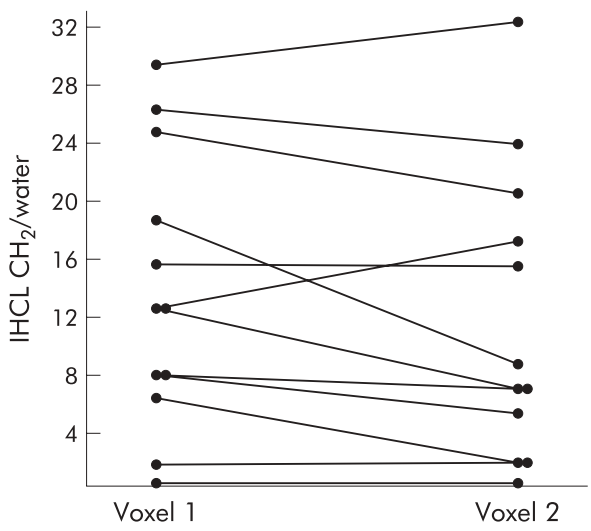

Figure 3 Difference in intrahepatocellular lipid $(\mathrm{IHCL})$ content between voxels placed in different positions in the left lobe of the liver.

was measured three times in a subset of 12 subjects; the mean $\mathrm{CoV}$ for measurement of liver volume was $1.16 \%$.

As expected, the volume of a subject's liver was related to IHCL content $(r=0.62, \mathrm{p}<0.01)$. There were also significant correlations between liver volume and body weight $(r=0.72$, $\mathrm{p}<0.01)$, BMI $(r=0.55, \mathrm{p}<0.01)$, intra-abdominal AT $(r=0.61, \mathrm{p}<0.01)$, non-abdominal internal AT $(r=0.44$, $\mathrm{p}=0.03)$, and intra-abdominal AT as a percentage of body weight $(r=0.40, \mathrm{p}=0.05)$.

\section{IHCL and whole body AT content}

A wide range of IHCL content was observed in our cohort of subjects. Examining the total group with respect to BMI, independent of whether they were in the NASH or control group, overweight and obese subjects generally showed significantly higher levels compared with lean subjects $\left(\right.$ BMI $>25 \mathrm{~kg} / \mathrm{m}^{2}(\mathrm{n}=23):$ GM 7.7 (IQR 4.0-28.6) $v$ BMI $<25 \mathrm{~kg} / \mathrm{m}^{2}(\mathrm{n}=11)$ : GM 1.3 (IQR 0.3-3.6); $\mathrm{p}=0.004$ ).

Multiple regression analysis revealed, after allowing for sex and age, a significant relationship between IHCL content of the liver and

(a) total body fat expressed as a percentage of body weight (IHCL increased by $22 \%$ for each $1 \%$ increase in AT $(95 \%$ confidence interval (CI) 10-36\%); $\mathrm{p}=0.001$ ),

(b) regional adiposity (IHCL increased by $21 \%$ for each $1 \%$ increase in total subcutaneous AT (95\% CI 6-38\%; $\mathrm{p}=0.007)$, and

(c) central obesity (IHCL increased by $72 \%$ for each $1 \%$ increase in subcutaneous abdominal AT (95\% CI 27$133 \%), p=0.001$, and IHCL increased by $104 \%$ for each

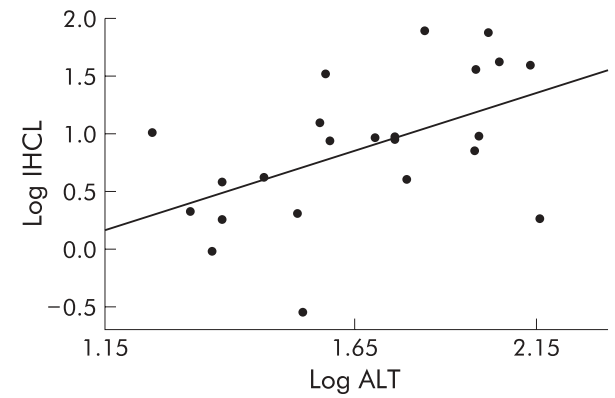

Figure 4 Relationship between intrahepatocellular lipid (IHCL) content and serum alanine aminotransferase (ALT): $\log _{\mathrm{e}} \mathrm{IHCL}=-3.271+1.3377$ $\times \log _{\mathrm{e}} \operatorname{ALT}(r=0.57, \mathrm{p}=0.006)$.
$1 \%$ increase in intra-abdominal AT (95\% CI 37-205\%), $\mathrm{p}=0.001)$.

Including an additional term for the NASH population did not significantly improve the fit of any of the multiple regression models.

\section{IHCL and IMCL content}

Multiple regression analysis showed no significant correlation between IHCL and intramyocellular lipids (IMCL) in the tibialis and soleus although there was a significant relationship between these fat depots (tibialis muscle, $\mathrm{p}=0.004$; soleus muscle, $\mathrm{p}=0.04$ ) and percentage body fat, after accounting for sex and age.

\section{IHCL and LFTs}

The relationship between IHCL and the results of LFTs was also investigated. Multiple regression analyses revealed a significant relationship between ALT and liver fat content $(r=0.57, \mathrm{p}=0.006)$ (fig 4$)$ but not between IHCL and either ALP or $\gamma$ GT.

\section{DISCUSSION}

This in vivo study aimed to evaluate the relationship between IHCL levels and body adiposity. The results show that measurable IHCL is present in healthy individuals with no history of liver function test abnormalities and no known history of metabolic or liver disease. We have found that IHCL is positively correlated to regional AT content. However, we found no correlation between IHCL content and IMCL content.

To our knowledge, this is the first systematic study of the relationship between IHCL level and body adiposity. In a previously published in vitro study of 107 unselected cadaver livers, Donhoffer detected IHCL in all subjects, with a triglyceride content ranging from $1.7 \%$ to $25.6 \%{ }^{29}$ They suggested that normal livers may contain as much as $5.5 \%$ triglyceride (wet weight) and that a triglyceride content greater than $8 \%$ may be a reliable sign of pathological fatty liver. ${ }^{29}$ In our in vivo study, we also found a wide range of hepatic fat content in the study population as a whole (GM 4.4 (IQR 1.3-12.4)), with resonances from hepatic triglycerides observable in all volunteers examined, even though they had no history of liver disease. Lower levels of IHCL $(<5 \%)$ were generally found in younger and healthier volunteers, while older less active subjects showed higher IHCL content.

Similar findings have been reported in a number of in vivo studies. Seppala-Lindroos and colleagues ${ }^{18}$ have reported a wide range of IHCL deposition in the general population using ${ }^{1} \mathrm{H}$ MRS. They subdivided their cohort of subjects into low IHCL content $(1.7 \pm 0.2 \%)$ and high IHCL content $(10.5 \pm 2.0 \%)$ although no clear physiological/biochemical rationale for this "cut off" was presented. The former group was associated with "healthier subjects", including significantly lower LFT and plasma lipids levels although there were no significant differences in BMI, percentage body fat, or $\mathrm{VO}_{2 \max }$. Tarasow and colleagues ${ }^{30}$ have also reported wide ranges of IHCL deposition by ${ }^{1} \mathrm{H}$ MRS in healthy volunteers, attributing this to a possible tendency to obesity, while Hwang and colleagues ${ }^{16}$ studied 15 non-diabetic subjects and found IHCL content of $10.9 \pm 7.2 \%$. In subjects with familial hypobetalipoproteinaemia, Schonfeld and colleagues ${ }^{31}$ found increased IHCL content compared with controls although the latter still showed considerable levels of hepatic triglycerides. Liver fat percentages were $16.7 \pm 11.5$ and $3.3 \pm 2.9$.

The geometric mean for IHCL content observed in the present study was similar (GM 4.4 (IQR 1.3-12.4)) to those quoted in the literature, supporting previous in vitro and in vivo findings that hepatic fat infiltration is widespread in the 
general population. ${ }^{16}{ }^{18}{ }^{29-31}$ Indeed, the IHCL levels measured in some of our volunteers were similar or higher than those in patients with biopsy proven NASH, suggesting that those individuals with biopsy proven hepatic steatosis/NASH represent "the tip of the iceberg" in epidemiological terms. Further work is however required to ascertain the "normal" level of IHCL in the general population and establish the levels at which triglyceride deposition becomes pathological.

Interestingly, our preliminary results showed a significant correlation between IHCL and ALT, but not $\gamma$ GT or ALP, in the study population as a whole. This is surprising as an elevated $\gamma \mathrm{GT}$ is generally thought to be a serum marker of hepatic steatosis with elevated transaminases considered by many to clinically represent hepatic inflammation in NASH.

Previous studies have produced conflicting results with regard to distribution of triglycerides in the liver. Kremer and colleagues, ${ }^{21}$ in a study of 29 human cadavers, found significant variation in the fat content across the liver. The authors showed that the degree of variation was greatest in subjects with the highest liver fat content. ${ }^{21}$ However, Gaal and Husveth, ${ }^{22}$ in a study of nine dairy cows, showed that the lipid content of a liver biopsy $(0.08-0.15 \mathrm{~g})$ was almost identical to that from a large (150-200 g) liver sample taken from the same animal. In vivo, Tarasow and associates ${ }^{30}$ have reported a variation of $6.5-87.4 \%$ in IHCL from two different positions in the liver in five subjects. Schonfeld and colleagues $^{31}$ have also reported considerable regional IHCL variation, as well as diurnal alteration in liver fat content, which emphasises the need for an accurate and reproducible protocol in serial studies. In our study we have shown that, although as a cohort no significant regional differences in IHCL levels could be observed, a small number of individuals showed large regional variations. This variation in IHCL content did not appear to be related to total body adiposity, liver size, or to depend on the amount of triglycerides present. At present, it is unclear what factors may determine and modulate IHCL accumulation in human subjects, both in health and disease, but may include genetic susceptibility. Further understanding of the possible heterogeneity of liver triglyceride distribution is important as it may have major implications for interventional studies.

The relationship between IHCL accumulation and whole body AT deposition and activity is unclear. In the present study, we have shown that there is a significant correlation between IHCL content and regional adiposity, after accounting for sex and age. The relationship between liver triglyceride content and adiposity suggests that IHCL accumulation may be influenced by AT deposition and/or activity, especially abdominal AT. Previous in vivo MRS and biopsy studies examining the relationship between liver fat and intra-abdominal AT in male and female subjects have produced conflicting results. Seppala-Lindroos and colleagues, ${ }^{18}$ using in vivo MRS, reported no correlation between intra-abdominal AT and hepatic fat in 30 healthy male subjects. Similar findings were reported by Tiikkainen and colleagues, ${ }^{32}$ who showed that during weight loss, decreases in IHCL were independent of changes in intra-abdominal and subcutaneous fat and that IHCL appears to be related to dietary fat content rather than the size of endogenous fat depots. Kral and colleagues ${ }^{33}$ also examined the relationship between adiposity and hepatic fat. However, using multiple regression analysis, they found that the waist to hip ratio accounted for the degree of steatosis, evaluated by biopsy, with weight, BMI, and body fat content having little influence, suggesting that abdominal AT content is the important factor in the development of fatty liver. Combining all of these data, a picture emerges where IHCL levels may not only reflect general adiposity but may be related to the level of metabolic activity of the various AT depots. ${ }^{34}$ Subcutaneous fat depots have been shown to have significantly lower blood flow and hormone sensitive lipase rate of action than abdominal AT. These functional differences may in part help to explain the large variability in IHCL content in subjects with a similar BMI and percentage body fat, as observed in our study.

Although the physiological mechanisms that mediate the relationship between adiposity and IHCL accumulation are yet to be identified, it is possible that levels of IHCL may reflect AT depot activity rather than simply reflecting the size of endogenous AT stores. Thus the high lipolytic activity of abdominal fat depots, rather than its size, may be responsible for IHCL accumulation in different individuals. ${ }^{35}$ Intraabdominal and abdominal subcutaneous AT may for example lead to increased portal flux of free fatty acids to the liver and increased circulating plasma triglycerides, respectively, both of which could lead to reduced lipolysis and increased IHCL stores. As free fatty acid flux from abdominal fat depots have also been shown to correlate with insulin resistance, ${ }^{2}$ this may represent a link between obesity, liver fat, and insulin resistance.

In summary, this study has shown that liver triglyceride deposition is closely related to abdominal AT content in subjects with biopsy proven NASH and in healthy subjects. The large individual variation may possibly reflect independent genetic/environmental factors. Our results also confirmed the ability of MRS to study the process of triglyceride deposition in the liver non-invasively. A combination of this technique and ${ }^{31} \mathrm{P}$ MRS, which gives a handle on cell membrane turnover and fibrosis, may help in the future to elucidate the underlying relationships associated with the progression from fat infiltration of the liver to NASH and cirrhosis. $^{36-39}$ MRS also holds the promise of being an invaluable in vivo tool in further human interventional and genetic studies. ${ }^{40} 41$

\section{ACKNOWLEDGEMENTS}

The authors would like to thank Professor GM Bydder and Dr W Curati, MRI Unit, Hammersmith Hospital, Imperial College London, for their help with this project. We also like to thank the British Medical Research Council (UK) for their financial support, including grant number G9900178.

\section{Authors' affiliations}

E L Thomas, G Hamilton, N Patel, R O'Dwyer, J D Bell, Robert Steiner MR Unit, Imaging Sciences Department, MRC Clinical Sciences Centre, Hammersmith Hospital, Imperial College London, London, UK

S D Taylor-Robinson, Division of Medicine (Medicine A), Faculty of Medicine, Hammersmith Hospital, Imperial College London, London, UK C J Doré, MRC Clinical Trials Unit, London, UK

R D Goldin, Histopathology Department, St Mary's Hospital, London, UK

Conflict of interest: None declared.

\section{REFERENCES}

1 Boden G. Role of fatty acids in the pathogenesis of insulin resistance and NIDDM. Diabetes 1997;46:3-10.

2 Boden G. Interaction between free fatty acids and glucose metabolism. Curr Opin Clin Nutr Metab Care 2002;5:545-9.

3 Marx J. Unravelling the causes of diabetes. Science 2002;296:686-9.

4 Yki-Jarvinen H. Ectopic fat accumulation: an important cause of insulin resistance in humans. J R Soc Med 2002;95:39-45.

5 Youssef W, McCullough AJ. Diabetes mellitus, obesity, and hepatic steatosis. Semin Gastrointest Dis 2002;13:17-30.

6 Chitturi S, Abeygunasekera S, Farrell GC, et al. NASH and insulin resistance: Insulin hypersecretion and specific association with the insulin resistance syndrome. Hepatology 2002:35:373-9.

7 Song S. The role of increased liver triglyceride content: a culprit of diabetic hyperglycaemia? Diabetes Metab Res Rev 2002;18:5-12.

8 Crowley H, Lewis WD, Gordon F, et al. Steatosis in donor and transplant liver biopsies. Hum Pathol 2000;31:1209-13.

9 Longo R, Ricci C, Masutti F, et al. Fatty infiltration of the liver. Quantification by ${ }^{\top} \mathrm{H}$ localized magnetic resonance spectroscopy and comparison with computed tomography. Invest Radiol 1993;28:297-302. 
10 Ricci C, Longo R, Gioulis $\mathrm{E}$, et al. Noninvasive in vivo quantitative assessment of fat content in human liver. J Hepatol 1997;27:108-13.

11 Thomsen C, Becker U, Winkler K, et al. Quantification of liver fat using magnetic resonance spectroscopy. Magn Reson Imaging 1994;12:487-95.

12 Longo R, Pollesello $P$, Ricci $C$, et al. Proton MR spectroscopy in quantitative in vivo determination of fat content in human liver steatosis. J Magn Reson Imaging 1995;5:281-5

13 Szczepaniak LS, Babcock EE, Schick F, et al. Measurement of intracellular triglyceride stores by ${ }^{1} \mathrm{H}$ spectroscopy: validation in vivo. Am J Physiol 1999;276:E977-89.

14 Anderwald C, Bernroider E, Krssak M, et al. Effects of insulin treatment in type II diabetic patients on intracellular lipid content in liver and skeletal muscle. Diabetes 2002;51:3025-32.

15 Carey DG, Cowin GJ, Galloway GJ, et al. Effect of rosiglitazone on insulin sensitivity and body composition in type II diabetic patients. Obes Res 2002; 10:1008-15.

16 Hwang JH, Stein DT, Balent L, et al. Simultaneous quantitative assessment of intraheaptic triglycerides and $\mathrm{IMCL}$ using ${ }^{1} \mathrm{H}$ MRS in non-diabetic subjects: relationship to insulin sensitivity. Proc Intl Soc Mag Reson Med 2002; 1:10.

17 Mayerson AB, Hundal RS, Dufour S, et al. The effects of rosiglitazone on insulin sensitivity, lipolysis, and hepatic and skeletal muscle triglyceride content in patients with type II diabetes. Diabetes 2002;51:797-802.

18 Seppala-Lindroos A, Vehkavaara S, Hakkinen AM, et al. Fat accumulation in the liver is associated with defects in insulin suppression of glucose production and serum free fatty acids independent of obesity in normal men. J Clin Endocrinol Metab 2002;87:3023-8

19 Tiikkainen M, Tamminen M, Hakkinen AM, et al. Liver-fat accumulation and insulin resistance in obese women with previous gestational diabetes. Obes Res 2002;10:859-67.

20 Simha V, Szczepaniak LS, Wagner AJ, et al. Effect of leptin replacement on intrahepatic and intramyocellular lipid content in patients with generalized lipodystrophy. Diabetes Care 2003;26:30-5.

21 Kremer GJ, Katzenbach G, Victor N. Regional distribution of the triglyceride content in the human liver. Verh Dtsch Ges Inn Med 1969;75:884-7.

22 Gaal T, Husveth F. Comparison of the liver biopsy sample and the "whole liver" in respect of lipid content and fatty acid composition of lipids. Acta Vet Hung 1983;31:51-6.

23 Brunt EM, Janney CG, Di Bisceglie AM, et al. Nonalcoholic steatohepatitis: a proposal for grading and staging the histological lesions. Am J Gastroenterol 1999;94:2467-74.

24 Saeed N. Magnetic resonance image segmentation using pattern recognition, and applied to image registration and quantitation. NMR Biomed 1998;11:157-67.

25 Vanhamme L, van den Boogaart A, Van Huffel S. Improved method for accurate and efficient quantification of MRS data with use of prior knowledge. J Magn Reson 1997;129:35-43.
26 Naressi A, Couturier C, Devos JM, et al. Java-based graphical user interface for the MRUI quantitation package. MAGMA 2001;12:141-52.

27 Rico-Sanz J, Thomas EL, Jenkinson G, et al. Diversity in levels of intracellular total creatine and triglycerides in human skeletal muscles observed by ${ }^{1} \mathrm{H}$ MRS. J Appl Physiol 1999;87:2068-72.

28 Thomas EL, Saeed N, Hajnal JV, et al. Magnetic resonance imaging of total body fat. J Appl Physiol 1998;85:1778-85.

29 Donhoffer H. Quantitative estimation of lipids in needle biopsy sized specimens of cadaver liver. Acta Med Acad Sci Hung 1974;31:47-9.

30 Tarasow E, Siergiejczyk L, Panasiuk A, et al. MR proton spectroscopy in liver examinations of healthy individuals in vivo. Med Sci Monit 2002;8:MT36-40.

31 Schonfeld G, Patterson BW, Yablonskiy DA, et al. Fatty liver in familial hypobetalipoproteinemia: triglyceride assembly into VLDL particles is affected by the extent of hepatic steatosis. J Lipid Res 2003;44:470-8.

32 Tiikkainen $M$, Bergholm R, Vehkavaara $S$, et al. Effects of identical weight loss on body composition and features of insulin resistance in obese women with high and low liver fat content. Diabetes 2003;52:701-7.

33 Kral JG, Schaffner F, Pierson RN Jr, et al. Body fat topography as an independent predictor of fatty liver. Metabolism 1993;42:548-51.

34 Tan GD, Goossens GH, Humphreys SM, et al. Upper and lower body adipose tissue function: a direct comparison of fat mobilization in humans. Obes Res 2004; 12:114-18.

35 Large V, Arner P. Regulation of lipolysis in humans. Pathophysiological modulation in obesity, diabetes, and hyperlipidaemia. Diabetes Metab 1998:24:409-18.

$36 \operatorname{Lim}$ AK, Patel N, Hamilton G, et al. The relationship of in vivo ${ }^{31} \mathrm{P} M R$ spectroscopy to histology in chronic hepatitis C. Hepatology 2003;37:788-94.

37 Taylor-Robinson SD, Sargentoni J, Bell JD, et al. In vivo and in vitro hepatic ${ }^{31} \mathrm{P}$ magnetic resonance spectroscopy and electron microscopy of the cirrhotic liver. Liver 1997; 17:198-209.

38 Menon DK, Sargentoni J, Taylor-Robinson SD, et al. Effect of functional grade and etiology on in vivo hepatic phosphorus-31 magnetic resonance spectroscopy in cirrhosis: Biochemical basis of spectral appearances. Hepatology 1995;21:417-27.

39 Taylor-Robinson SD, Sargentoni J, Bell JD, et al. In vivo and in vitro hepatic phosphorus-31 magnetic resonance spectroscopy and electron microscopy in chronic ductopenic rejection of human liver allografts. Gut 1998;42:735-43

40 Bell JD, Taylor-Robinson SD. Assessing gene expression in vivo: magnetic resonance imaging and spectroscopy. Gene Therapy 2000;7:1259-64.

41 Bhakoo KK, Bell JD, Cox IJ, et al. The application of magnetic resonance imaging and spectroscopy to gene therapy. Methods Enzymol 2004;386:303-13

\section{EDITOR'S QUIZ: GI SNAPSHOT}

\section{Oesophageal ulceration: more than meets the naked eye?}

Robin Spiller, Editor

\section{Clinical presentation}

A 56 year old male presented with an eight week history of dyspepsia, dysphagia, and epigastric tenderness. Symptoms were controlled on a trial of proton pump inhibitor (lansoprazole) but recurred when this was discontinued. He also had a history of asthma, with numerous admissions to hospital due to bronchospasm. His medications at presentation included fluticasone, ipratropium, and salbutamol inhalers along with monteleukast and theopylline. He was

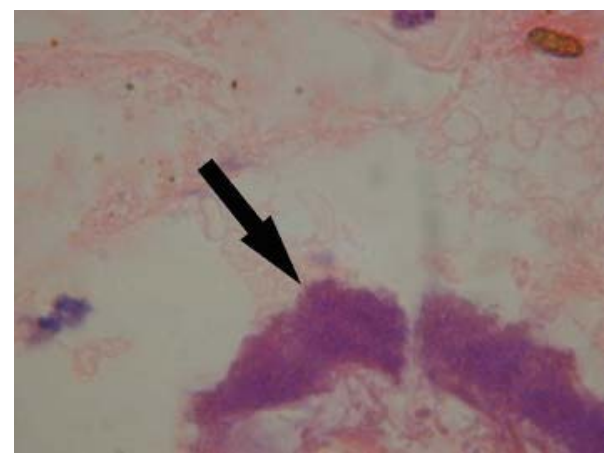

Figure 1 Oesophageal biopsy. a non-smoker, was not diabetic, and had no other documented illnesses. Endoscopy revealed a focal area of ulceration in the lower third of the oesophagus, in addition to more extensive confluent ulceration in the middle third. There was also duodenitis and ulceration of the first part of the duodenum. The Clo test was negative. Oesophageal biopsies (fig l) confirmed ulceration of the oesophagus and revealed acute on chronic inflamed granulation tissue.

\section{Question}

What is the cause of this man's oesophageal ulceration? See page 133 for answer

This case is submitted by:

J W Cash Royal Victoria Hospital, Belfast, Northern Ireland, UK C McConville Lagan Valley Hospital, Lisburn, Northern Ireland, UK D O'Rourke Belfast City Hospital, Northern Ireland, UK T Harding Department of Medicine, Lagan Valley Hospital, Lisburn, Northern Ireland, UK

Correspondence to: Dr J Cash, Department of Medicine, Royal Victoria Hospital, Grosvenor Rd, Belfast BT12 6BA, UK; w.cash@qub.ac.uk 